\title{
Long- and medium-range components of the nuclear force in quark-model based calculations
}

\author{
D. Hadjimichef, ${ }^{1}$ J. Haidenbauer, ${ }^{2}$ and G. Krein ${ }^{3}$ \\ ${ }^{1}$ Departamento de Física, Universidade Federal de Pelotas, 96010-900 Pelotas, RS, Brazil \\ ${ }^{2}$ Forschungszentrum Jülich, Institut für Kernphysik, D-52425 Jülich, Germany \\ ${ }^{3}$ Instituto de Física Teórica, Universidade Estadual Paulista, Rua Pamplona, 145-01405-900 São Paulo, SP, Brazil
}

(Received 16 October 2000; published 14 February 2001)

\begin{abstract}
Quark-model descriptions of the nucleon-nucleon interaction contain two main ingredients, a quarkexchange mechanism for the short-range repulsion and meson exchanges for the medium- and long-range parts of the interaction. We point out the special role played by higher partial waves, and in particular the ${ }^{1} F_{3}$, as a very sensitive probe for the meson-exchange part employed in these interaction models. In particular, we show that the presently available models fail to provide a reasonable description of higher partial waves and indicate the reasons for this shortcoming.
\end{abstract}

DOI: $10.1103 /$ PhysRevC.63.035204

PACS number(s): 21.30. $-\mathrm{x}, 13.75 . \mathrm{Cs}, 24.85 .+\mathrm{p}, 12.39 . \mathrm{Jh}$

\section{INTRODUCTION}

The traditional and most accurate description of the nucleon-nucleon $(N N)$ force at low energies is based on meson-exchange models. There are many versions of such models in the literature (cf., e.g., Refs. [1,2] for a short historical view and many references). With almost no exception, the various models have the common feature that the long-range part of the interaction is described by one-pion exchange (OPE) and the medium-range part is described by contributions from two-pion exchange, usually parametrized in terms of the $\rho$ and $\sigma$ mesons. On the other hand, the treatment of the short-range part of the interaction can differ considerably among the $N N$ models. This part is assumed to receive contributions from multimeson exchanges. At very short distances the interaction is either parametrized phenomenologically or regularized by means of vertex form factors. Those parametrizations or form factors are expected to be explained ultimately by invoking quark-gluon degrees of freedom.

However, direct use of the QCD Lagrangian (or Hamiltonian) for studying processes at the nuclear scale has been so far possible only in large-scale numerical simulations on a supercomputer. The use of a quark model seems therefore necessary for analytical calculations. Unfortunately, the formulation of an accurate and, at the same time, sufficiently simple quark model is very difficult, for several reasons. Perhaps the most notorious obstacle is our difficulty in identifying the relevant effective degrees of freedom that operate at the confinement scale. Despite this, a large body of hadronic spectroscopic and strong-decay data can be described reasonably well by the constituent quark model (CQM) [3]. In the $\mathrm{CQM}$, the low-energy spectrum of QCD is postulated to be built from spin-1/2 colored constituent massive quarks, which are confined within hadrons and interact weakly through one-gluon exchange (OGE).

Motivated by its simplicity and relative success in describing the data, many authors have used the CQM to study the short-range part of the $N N$ interaction in terms of OGE, using different approaches for the motion of the six-quark system. In such schemes, the $N N$ repulsion at short distances is generated dominantly by the quark Pauli exclusion prin- ciple and the color hyperfine interaction of the OGE. The initial works were based on adiabatic approximations of the Born-Oppenheimer type. The work of Liberman is the first along these lines [4], followed by the ones by Neudatchin $e t$ al. [5] and Harvey [6]. Beyond the adiabatic approximation, the resonating group method (RGM) has been widely used. Here the pioneering works stem from Ribeiro [7], Warke and Shankar [8], Oka and Yazaki [9], and Faessler et al. [10].

A common characteristic of these calculations is that they are unable to describe the qualitative features of the longand medium-range parts of the $N N$ interaction. In particular, they fail to describe the observed spin-orbit splitting of the spin-triplet $P$-wave phase shifts. In order to accommodate these features, meson exchanges and/or phenomenological potentials are added to the OGE. First of all the OPE interaction is taken into account. In addition, some mediumranged attractive contributions are supplemented. For example, in the works of the Tübingen-Salamanca (TUEBSAL) $[11,12]$ and the Salamanca-Valencia (SAL-VAL) [13] groups the exchange of a $\sigma$ meson is introduced. The model developed by the Tokyo group (TOK) [14] contains, besides $\pi$ and $\sigma$ exchange, an additional attractive phenomenological potential with different strength for each spin-isospin channel. In the model of the Kyoto-Niigata group (KYONII) [15], in addition to $\pi$ and $\sigma$, all other members of the scalar and pseudoscalar SU(3) meson nonets are included in an attempt to describe simultaneously nucleon-nucleon and hyperon-nucleon data. A common characteristics of these models is that vector-meson exchanges $(\omega, \rho)$ are not considered, the rationale for this being that the interactions generated by $\omega$ and $\rho$ exchanges are presumed to be of very short range and therefore their effects should be more appropriately taken into account by a quark-exchange mechanism. Another reason for leaving the vector mesons out is that the strong central repulsion originating from the time component of the vector meson exchange between quarks of different nucleons provides contributions qualitatively similar to the ones provided by the quark-exchange mechanism, and simultaneous consideration of both contributions would therefore lead to double counting [16]. Although the long-range part generated by the space components of vector meson exchange has no counterpart in the quark-exchange mecha- 
nism, the explicit realization of this component in a quark model is a nontrivial task and has not been fully realized (see, e.g., Ref. [17] for an initial attempt in this direction).

In all these approaches the additional parameters, such as meson-quark coupling strengths and form factors, are adjusted in part by a fit to the low $N N$ partial waves, i.e., those partial waves that are mostly sensitive to the short-range part of the $N N$ interaction. In general, the resulting description of the $N N$ phase shifts, in particular of the $S$ and $P$ waves, is very impressive. This is certainly an achievement because it is important to realize that the calculations are heavily constrained by the requirement that the added interactions still give a decent description of the mass splittings of the lowlying baryonic spectrum. This remark is particularly relevant for those approaches where the meson-exchange pieces contribute also to isolated baryons $[11-13,15]$ and not only to the $N N$ interaction [14].

Higher partial waves are predominantly determined by the longer-ranged pieces of the $N N$ force. These partial waves are usually not considered in the fitting procedure and therefore the corresponding results can be regarded as genuine predictions. In particular, this means that those higher partial waves are a good testing ground for the reliability of the medium- and long-range components employed in those quark models of the $N N$ interaction. In practice, however, the predictions of quark models for higher partial waves are rarely displayed. There are only a few works where the authors present phase shifts for $F$ [15] or even $G$ waves [14]. Indeed the results are not very encouraging. They reveal striking differences not only from phase-shift analyses but also from the phases predicted by conventional mesonexchange models of the $N N$ interaction.

In the present paper we want to investigate the origin of these differences. Specifically we want to examine the ingredients that constitute the medium- and long-range pieces of quark models and compare them with those used in conventional meson-exchange models. Thereby we aim at a qualitative appreciation of the reasons for the observed failure in describing the higher $N N$ partial waves in terms of the dynamics on which those quark models are based. Thus, our study is complementary to a recent investigation carried out by the Paris group [18]. In this work $N N$ observables were calculated with a model built from the core (short-range) part of the quark model of the Tokyo group [14] and supplemented, at intermediate and long internucleonic distances, by the $N N$ forces generated from the Paris potential [19]. It was found that such an approach leads to a very poor description of the data, with $\chi^{2}$ /data ranging from 20 to 160 .

Our paper is organized as follows. In the next section we review shortly the ingredients of those quark models of the $N N$ interaction that we consider in our investigation. Furthermore, we argue and establish via sample calculations that the $F$ and $G$ waves are not sensitive anymore to the shortrange part of the $N N$ force, governed by quark-exchange mechanisms, and therefore are very well suited for testing the medium-range pieces that are employed in present-day quark models. In Sec. III we compare the predictions of specific quark models for the ${ }^{1} F_{3}$ and ${ }^{1} G_{4}$ partial waves with those of a simple conventional one-boson-exchange model of the $N N$ force. In addition we carry out a detailed analysis of the behavior of the corresponding potentials for internucleonic distances around $1 \mathrm{fm}$ in order to understand the dynamical origin of the differences that we observe in the phase-shift results. The paper ends with a general discussion about possible origins of the failure of quark models in describing those higher partial waves. Furthermore, suggestions on a different strategy to study the short-range part of the $N N$ force as derived from subnucleonic degrees of freedom are given.

\section{QUARK EXCHANGE AND HIGHER $N \boldsymbol{N}$ PARTIAL WAVES}

The medium-range parts of models for the $N N$ forces can be investigated most efficiently by looking at higher partial waves of the $N N$ interaction [1]. For orbital angular momenta $L \geqslant 3$ ( $F, G$, etc., waves) the centrifugal barrier is, in general, already sufficiently large to suppress contributions from the short-range part of the $N N$ interaction, specifically from quark-exchange processes, as we will show below. Furthermore, it is preferable to look at spin-singlet partial waves because here the strong tensor force from the OPE is absent and possible spin-orbit forces cannot contribute either. These contributions to the $N N$ interaction are not relevant for the points we want to address. From those considerations it follows that the ${ }^{1} F_{3}$ should be the best candidate for testing models for the medium-range interaction and most of our study will concentrate on this partial wave. However, we will look at the ${ }^{1} G_{4}$ as well.

Our aim in this section is to demonstrate explicitly that the $F$ waves are indeed relatively insensitive to the shortranged pieces of the $N N$ interaction, i.e., those that involve quark exchanges between the nucleons. For that purpose we solve the scattering equation (Schrödinger equation) for some quark models using, however, only the part of the effective $N N$ interaction without the pieces that involve quark exchange and compare the resulting phase shifts with those obtained for the complete $N N$ interaction model that include quark exchange. Specifically, we solve

$$
\left[-\frac{\boldsymbol{\nabla}^{2}}{M}+V_{N N}^{D}(\boldsymbol{r})\right] \psi(\boldsymbol{r})=E \psi(\boldsymbol{r})
$$

where $M$ is the nucleon mass, $E$ is the two-nucleon relative energy, and $V_{N N}^{D}$ is the "direct" effective $N N$ interaction kernel. The "exchange" contribution to the effective $N N$ interaction is neglected.

In the case of the TOK potential, $V_{N N}^{D}(\boldsymbol{r})$ is the effective meson-exchange potential (EMEP) $\bar{V}^{\text {EMEP }}$ whose explicit form is given by Eqs. (16)-(26) in Ref. [14]. It contains contributions from the OPE from a $\sigma$-like part and from an attractive phenomenological central and spin- and isospindependent potential of Gaussian form. Note that the $\pi$ and $\sigma$ exchanges take place between the quarks. The corresponding contribution to $V_{N N}^{D}(\boldsymbol{r})$ is the Fourier transform of the convolution of the microscopic quark-quark interaction $V_{q q}(\boldsymbol{q})$ and the nucleon form factor $F(\boldsymbol{q})$ at each vertex: 


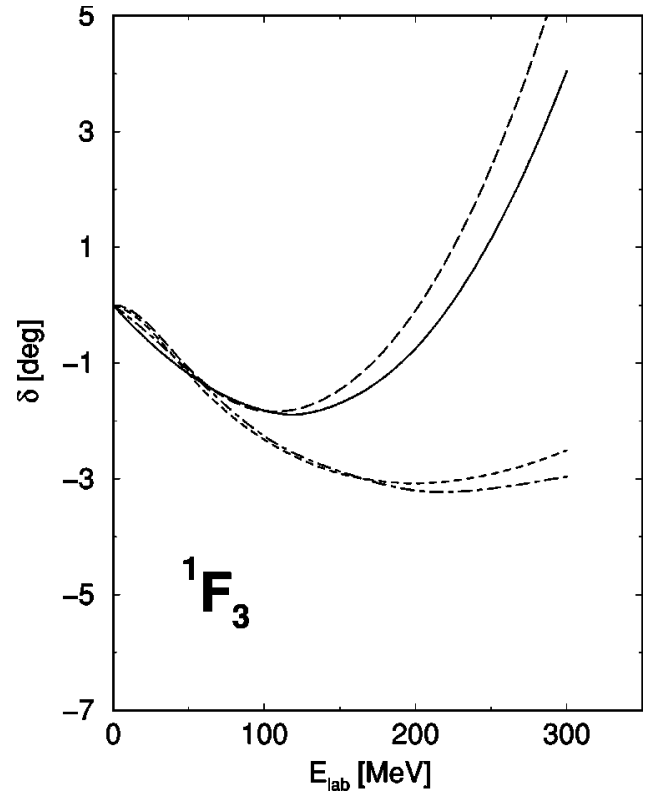

FIG. $1 .{ }^{1} F_{3}$ phase shift. Comparison of the results of the Tokyo [14] (solid line) and Kyoto [15] (dash-dotted line) groups based on the full model with our calculation (dashed curves) in which only the "direct'" part of the effective $N N$ interaction is employed; cf. Sec. II.

$$
V_{N N}^{D}(\boldsymbol{r})=\int \frac{d \boldsymbol{q}}{(2 \pi)^{3}} e^{i \boldsymbol{q} \cdot \boldsymbol{r}} F(\boldsymbol{q}) V_{q q}(\boldsymbol{q}) F(\boldsymbol{q})
$$

cf. their Eq. (19). We also want to mention that their pionexchange contribution contains a quadratic spin-orbit term of the form $-\bar{V}_{Q S O} \boldsymbol{\sigma} \cdot \boldsymbol{\sigma} \boldsymbol{L}^{2}$ [cf. Eqs. (18) and (25) of [14]], which does not vanish for singlet states. Our calculations are based on the model $Q$ as specified in Table 2 of Ref. [14].

The KYO-NII potential contains $\pi$ exchange as well as the exchange of two scalar [SU(3) flavor-singlet and -octet] mesons. All mesons are exchanged between the quarks. The quark-quark interactions $V_{q q}$ are simply the standard oneboson-exchange potentials for the $\pi$ and scalar mesons, respectively. The effective meson-exchange potential $V_{N N}^{D}(\boldsymbol{r})$ is obtained via a convolution according to Eq. (2). In our calculation we employ the model FSS as specified in Table III of Ref. [15].

The TUEB-SAL potential includes the $\pi$ and $\sigma$ mesons; both are exchanged between the quarks. The explicit form of their quark-quark interactions can be found, e.g., in Ref. [12]. The effective meson-exchange potential $V_{N N}^{D}(\boldsymbol{r})$ is again obtained via a convolution according to Eq. (2). Our calculations are based on the model parameters that were employed in Ref. [12].

Results for the ${ }^{1} F_{3}$ phases are presented in Fig. 1. The solid and dash-dotted lines show the phase shifts of the complete calculation with the TOK and KYO-NII potentials, respectively, taken from the original works $[14,15]$. The dashed curves are corresponding results obtained by us. As mentioned above, in our calculation only the medium- and long-range parts of these potentials were taken into account.

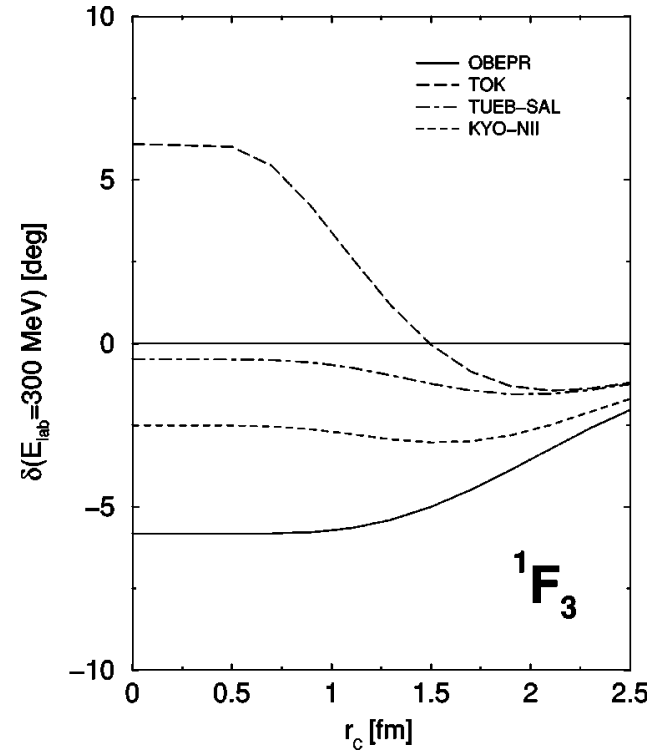

FIG. 2. ${ }^{1} F_{3}$ phase shifts at $E_{l a b}=300 \mathrm{MeV}$ as a function of the cutoff radius $r_{c}$ for the one-boson-exchange model OBEPR [1] (solid line) and the quark models of the Tokyo [14] (long-dashed line), Tübingen-Salamanca [12] (dash-dotted line), and KyotoNiigata [15] (short-dashed line) groups.

Short-range contributions from the quark-exchange processes were omitted. Evidently, the differences between the two calculations are fairly small, which means that the ${ }^{1} F_{3}$ phase shift is indeed primarily determined by the mediumand long-range parts of the $N N$ interaction. The quarkexchange part has definitely still an influence on this phase, but only in a quantitative sense and not on its qualitative behavior.

Note that we have carried out similar calculations also for other quark models of the $N N$ interaction such as the TUEBSAL and SAL-VAL potentials. Specifically, for the TUEBSAL model phase-shift results were provided privately to us by one of the authors of Ref. [12] and we could check explicitly that also in this case our results agree well with theirs.

In order to substantiate our conjecture that the $F$ waves are rather insensitive to the short-range part of the $N N$ interaction we designed a further test. We apply a cutoff of the form

$$
f(r)=\frac{1}{\left[1+\left(r_{c} / r\right)^{10}\right]}
$$

to the $N N$ potential $V_{N N}^{D}$. This cutoff function acts like a step function, such that for distances $r$ smaller than $r_{c}, f(r)$, and therefore the $N N$ potential, is practically zero. Then we insert this modified potential into the Schrödinger equation, calculate the phase shifts at a fixed energy, and study their dependence on the cutoff radius $r_{c}$. Corresponding results for the ${ }^{1} F_{3}$ partial wave at $E_{\text {lab }}=300 \mathrm{MeV}$, based on several $N N$ interaction models, are shown in Fig. 2 as a function of the cutoff radius $r_{c}$. One sees that the results for this partial wave are, in general, rather insensitive to the cutoff 
radius - and accordingly to the $N N$ interaction-for values of $r_{c}$ smaller than $r_{c} \approx 1 \mathrm{fm}$. Only in the case of the TOK potential is there a somewhat larger sensitivity resulting in deviations of the order of $10 \%$ already for $r_{c} \approx 0.7 \mathrm{fm}$.

Similar features were found also for the ${ }^{3} F_{3}$ partial wave. For $G$ waves (and in particular the ${ }^{1} G_{4}$ ) it turned out that the phase shifts are even insensitive to the $N N$ interaction for internucleon distances up to $r_{c} \approx 1.5 \mathrm{fm}$.

Let us come back to Fig. 2 again. With increasing cutoff radius $r_{c}$ much of the medium-range contributions will be suppressed as well and only the long-range part will be left, which is in the case of the ${ }^{1} F_{3}$ the spin-spin part of the OPE. Its contribution is present in all considered $N N$ potentials and therefore the phase-shift results should all converge to a common value for increasing values of $r_{c}$. However, even at the highest value shown in Fig. 2, $r_{c}=2.5 \mathrm{fm}$, there are still descrepancies. They are partly due to differences in the pion coupling constant and regularization schemes employed in the considered $N N$ models. But primarily they indicate that the medium-range part of those $N N$ interaction models is still sizable, even at internucleonic distances $r \approx 2.5 \mathrm{fm}$.

\section{MEDIUM-RANGE MESON EXCHANGE AND HIGHER $N N$ PARTIAL WAVES}

Having established the insensitivity of $F$ and higher partial waves to the quark-exchange part of the effective $N N$ interaction, we examine in this section the performance of the different quark models in describing these phase shifts. Furthermore, we scrutinize the dynamical ingredients that constitute the medium-range part of those interaction models. Specifically, we analyze the features of these potentials in $r$ space and we compare them with conventional mesonexchange models of the $N N$ interaction. For the latter we take the $r$-space version (OBEPR) of the Bonn $N N$ model [1]. There are certainly much more refined $N N$ models in the literature - in terms of the dynamical input (e.g, the full Bonn model [1]) as well as with regard to the description of $N N$ phase shifts $[20,21]$. However, for the qualitative comparison that we have in mind we need a model that has practically no nonlocalities and therefore is easy to handle in $r$ space. Furthermore, the Bonn OBEPR model includes all the one-boson-exchange contributions $(\pi, \rho, \omega, \sigma, \ldots$, exchanges) that are usually present in meson-exchange models and, most importantly, yields a fair description of the higher partial waves that we want to study. Therefore, the model OBEPR is indeed very well suited for our purpose.

In Figs. 3 and 4 we show results for the ${ }^{1} F_{3}$ and ${ }^{1} G_{4}$ waves, respectively, as a function of the $N N$ laboratory energy. The data points are taken from the phase-shift analyses of Refs. [22-24]. Evidently, the ${ }^{1} F_{3}$ phase shifts predicted by the quark models differ significantly from the one of the conventional meson-exchange model OBEPR; cf. Fig. 3. Specifically, the latter provides a reasonable description of this partial wave whereas the quark models deviate strongly from the experimental results. In fact, the KYO-NII potential is at least still in qualitative agreement with the data whereas the TOK potential yields completely unrealistic results. The predictions of the latter even change sign at higher energies.

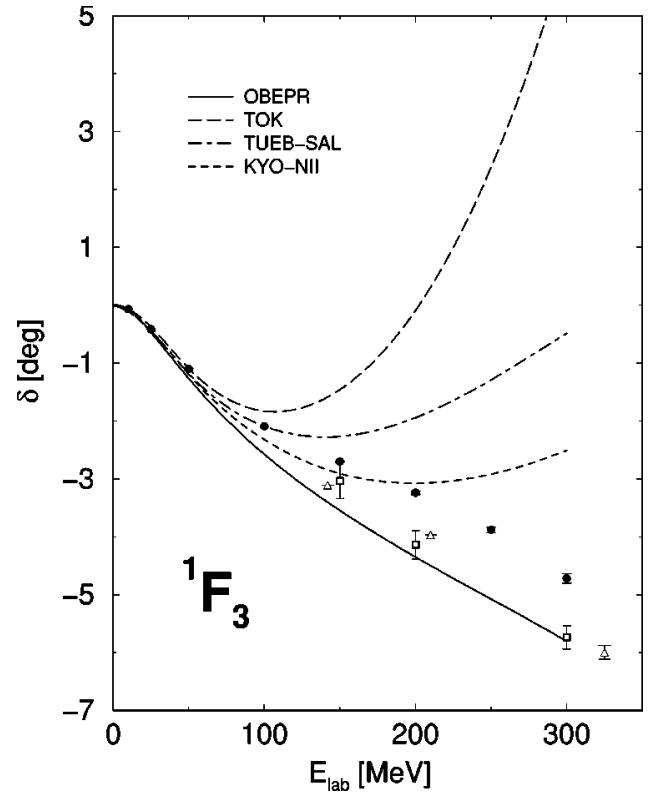

FIG. 3. ${ }^{1} F_{3}$ phase shifts predicted by the considered potential models. Same description of curves as in Fig. 2. Experimental phase shifts are from the analyses of the Nijmegen group [22] (solid circles), Arndt et al. [23] (squares), and Bugg and Bryan [24] (triangles).

Indeed all quark-model results show an upwards rising of the ${ }^{1} F_{3}$ phase shift for higher energies. This indicates that the medium-range part of all these models is too attractive.

In order to get a deeper understanding of the phase-shift results let us examine the different quark-model potentials in coordinate space. Corresponding graphs are presented in Fig. 5 for the ${ }^{1} F_{3}$ partial wave. Note that the curves do not include the contributions from the spin-spin part of the pion exchange. These are practically the same in all considered

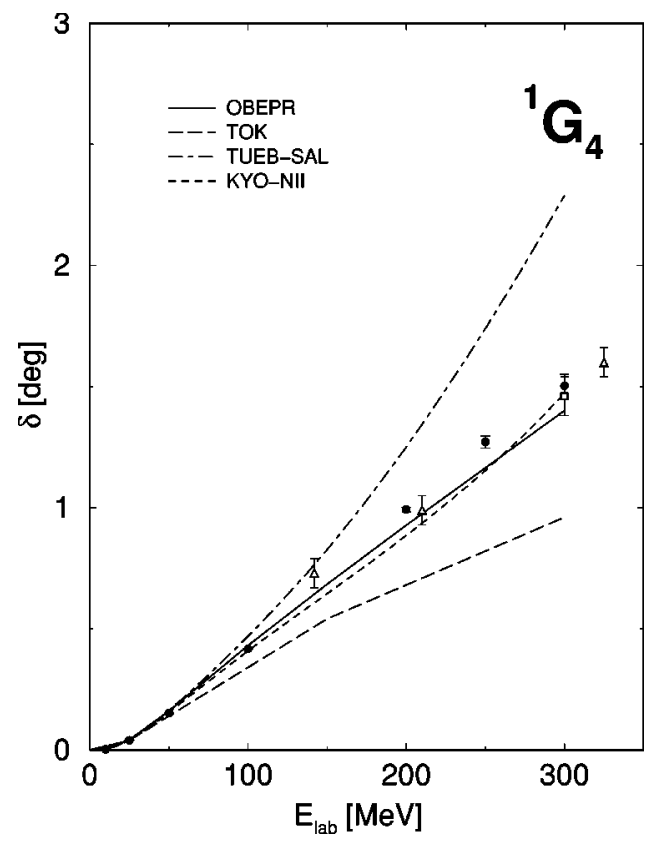

FIG. 4. ${ }^{1} G_{4}$ phase shifts. Same description as in Fig. 3. 


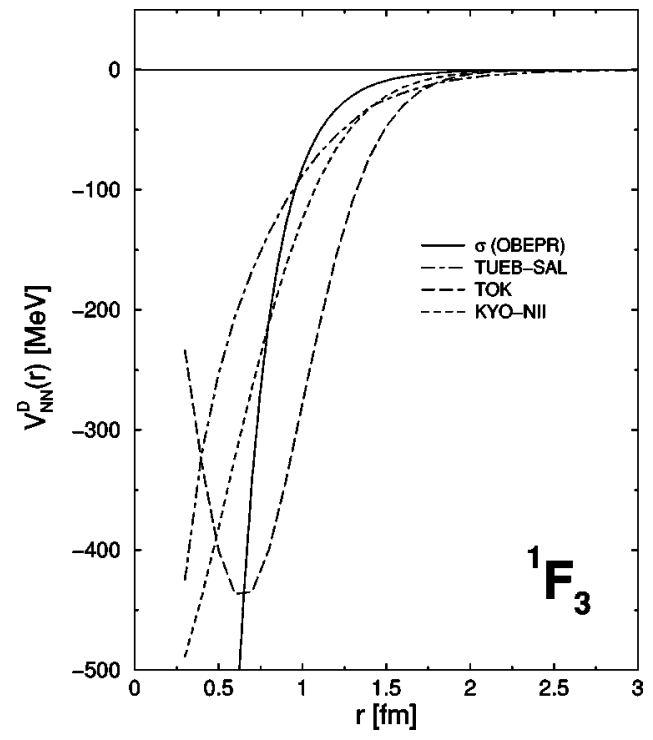

FIG. 5. "Direct'" effective $N N$ interaction of the quark models in the ${ }^{1} F_{3}$ partial wave. Note that the spin-spin part of the onepion-exchange contribution is omitted. Same description of curves as in Fig. 2. The solid line shows the $\sigma$-exchange contribution of the Bonn OBEPR model.

$N N$ models and therefore not interesting. Thus, Fig. 5 displays the "true" medium-range part of the quark models. As discussed in the previous section, this part is generated by $\sigma$ exchange and/or by $\sigma$-like phenomenological terms. Accordingly, we expect that it should correspond roughly to the $\sigma$-exchange contribution that is present in conventional OBE models. However, a comparison with the $\sigma$ exchange of the Bonn OBEPR model (cf. the solid line in Fig. 5) reveals that the latter is significantly smaller than the corresponding pieces in the quark models-for internucleonic distances $r$ $\geqslant 1 \mathrm{fm}$ relevant for the ${ }^{1} F_{3}$ partial wave. As a matter of fact, the medium-range part in the quark models is not only larger but seems to be longer ranged as well. In particular, the $\sigma$-like piece of the TOK potential (dashed curve) turns out to be exceptionally large. In view of this it is not surprising that the corresponding phase shifts deviate so strongly from the experimental results. On the other hand, the KYONII model, which comes closest to $\sigma$ exchange in the OBE model, gives also the best results for ${ }^{1} F_{3}$ among the quark models.

At this point let us recall that conventional mesonexchange models such as the OBEPR contain further ingredients that contribute to the potential at medium-range distances, namely, exchanges of the vector mesons $\rho$ and $\omega$. (Note that the OBEPR contains also contributions from $\eta$ and $a_{0}$ exchanges. However, their effect on the higher partial waves that we discuss here is negligibly small and therefore we do not consider them explicitly.) As mentioned already above, in the quark models of the $N N$ interaction contributions from vector-meson exchange are left out altogether, as is argued, for conceptional reasons [16]. Repulsive contributions, provided in conventional meson-exchange models predominantly by $\omega$ exchange, are present in the quark models too. Here they are generated, in general, by OGE in conjunc-

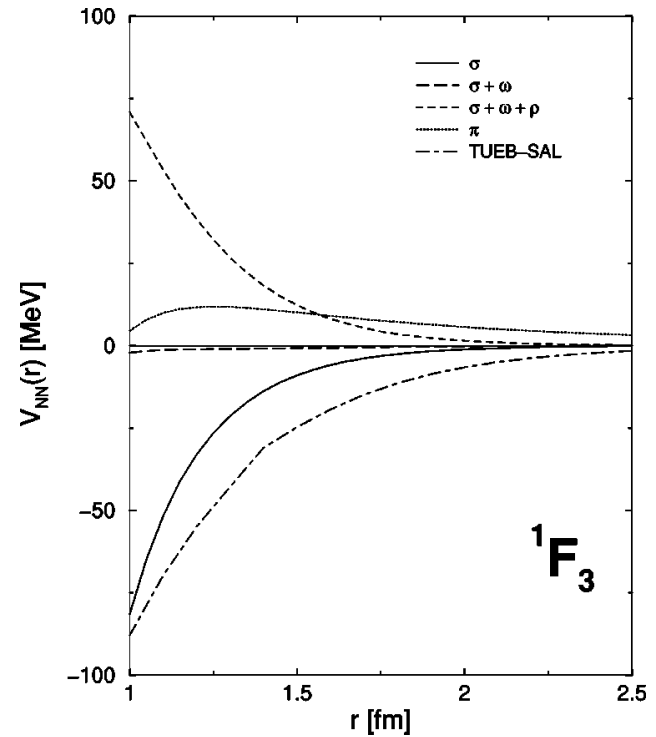

FIG. 6. Contributions to the potential in the ${ }^{1} F_{3}$ partial wave for the one-boson-exchange model OBEPR. $\sigma$ exchange, solid line; $\sigma$ $+\omega$ exchange, long-dashed line; $\sigma+\omega+\rho$ exchange, short-dashed line; $\pi$ exchange, dotted line. The dash-dotted curve shows the "direct", effective $N N$ interaction of the Tübingen-Salamanca model [12]; cf. Fig. 5.

tion with quark exchange between the nucleons. However, this mechanism is rather short ranged and therefore does not contribute to $F$ and higher partial waves anymore, as we have shown in the last section. Consequently, for the quark models the $\sigma$-like contributions shown in Fig. 5 constitute already the complete potential for medium-range distances. In conventional meson-exchange models such as the OBEPR the situation is different, as can be seen in Fig. 6. In this figure we show the potential resulting from $\sigma$ exchange (solid line) and then add consecutively the contributions from $\omega$ and $\rho$ exchange. The $\omega$-meson exchange practically cancels the attractive contribution from the $\sigma$ meson (longdashed line). Adding the $\rho$ meson (which is also repulsive in this partial wave) leads to a final result for the medium-range contributions which is repulsive (short-dashed line). The spin-spin part of the OPE-indicated by the dotted line-is repulsive as well. Combining those two leads to a strongly repulsive potential that produces phase shifts as required by the data. In the case of the quark models the complete medium-range contributions are always attractive; cf. Fig. 5 (the result for the TUEB-SAL model is also shown in Fig. 6 for ease of comparison). Thus, they will reduce the repulsion provided by the pion-exchange tail instead of enhancing it. In fact, for all models the attraction increases rather strongly when going to shorter distances and, consequently, eventually the whole potential becomes attractive. This feature is reflected in the behavior of the phase-shift results-which all turn to positive values for higher energies.

We consider the above results as evidence that vector mesons still play an important role in the $N N$ interaction at medium-range distances. Present-day quark-model descriptions lack contributions of the range and strength as provided by the $\omega$ and $\rho$ mesons in OBE models. 


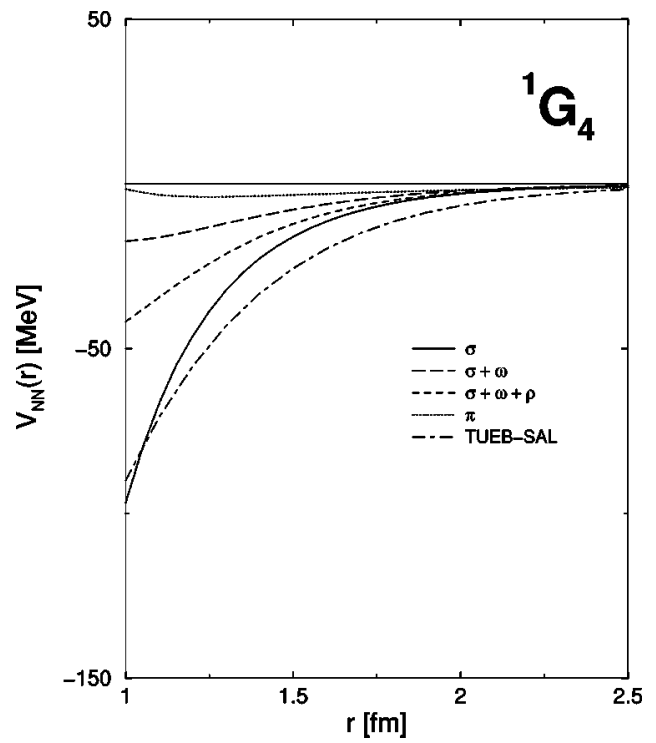

FIG. 7. Same as in Fig. 6 for the ${ }^{1} G_{4}$ partial wave.

Let us now look at the situation for the ${ }^{1} G_{4}$ partial wave. Corresponding results are shown in Fig. 4. Obviously, besides the OBEPR also the quark model KYO-NII is in good agreement with the phase-shift analysis. The other quark models either overshoot the experimental data (TUEB-SAL) or yield an underestimation (TOK). Also here it is instructive to look at the various contributions to the potential, which is done in Fig. 7. Again, we see that the medium-range component of the quark model (TUEB-SAL, dash-dotted line) is stronger and longer ranged than the $\sigma$-exchange contribution in the OBE potential (solid line). Moreover, in the OBE model there is again a non-negligible contribution from exchange of vector mesons. However, since the ${ }^{1} G_{4}$ partial wave is in a different isospin channel, now the contributions from the isovector mesons $(\rho, \pi)$ have the opposite sign. As a consequence the potential resulting from the $\omega$ exchange cancels to a large extent with the one resulting from the $\rho$ exchange. Thus, the total medium-range contributions are pretty close to the contributions of the $\sigma$ exchange alone (cf. the short-dashed and solid lines). This fact-that the contributions of the vector-meson exchange basically cancel out in this particular partial wave-is certainly responsible for the good performance of some quark models, specifically of the model KYO-NII. In the case of the TUEB-SAL model the $\sigma$-exchange contribution is simply too strong and long ranged and therefore the phase shifts are too large. For the TOK model the situation is somewhat different. The $\sigma$-like component of this potential has a phenomenological part whose parameters are adjusted for each of the four spinisospin $(S, T=0,1)$ channels separately; cf. Sec. 2.3. of Ref. [14] for details. For the ${ }^{1} F_{3}$ partial wave $[(0,0)$ channel] this phenomenological piece is rather strong as we have seen above whereas for ${ }^{1} G_{4}[(0,1)$ channel] it is much weaker. In addition, the TOK model contains a quadratic spin-orbit term of the form $-\bar{V}_{Q S O} \boldsymbol{\sigma} \cdot \boldsymbol{\sigma} \boldsymbol{L}^{2}$, which provides strong repulsion in singlet states with high orbital angular momentum $L$ such as the ${ }^{1} G_{4}$.

\section{DISCUSSION AND CONCLUSION}

In the last section we have seen that many of the presently available quark models of the $N N$ interaction have serious deficiencies in the description of higher partial waves. Specifically, we have shown that those models provide, in general, much too attractive forces at larger internuclear distances. A first possible and plausible explanation for these deficiencies was presented by Holinde several years ago [26]. He argued that the defect of those quark models might result from the fact that the entire repulsive contributions are generated by gluon exchange alone and, therefore, are of extremely short-ranged nature. As a remedy he advocated that at least part of the long-range tail of the $\omega$ exchange from the standard meson-exchange picture should be kept in those quark models.

Our detailed investigations suggest that the above conjecture is only one part of the truth. We confirmed that the repulsion provided by the quark models is much too short ranged and therefore does not affect the higher partial waves anymore as it would be required for a proper description of the corresponding phase shifts. However, the situation is more complex. We found evidence that, besides the $\omega$ exchange, also the long-range tail of the $\rho$ meson exchange is still felt by the $F$ and $G$ waves and therefore needed for a quantitative reproduction of those phases. As already pointed out above, contributions from those vector mesons are left out in the quark models from the very beginning - and there are no mechanisms in those models that would generate forces with similar features and comparable range. Finally, and most disturbingly, we found that most of the quark models contain attractive $(\sigma$-like) contributions that are rather strong and also rather long ranged.

The reason why such strong attractive forces need to be introduced in the quark models would require a thorough analysis of the short-range part of those models which is beyond the scope of the present study. Therefore, here we restrict ourselves to a plausible speculation that certainly deserves further detailed study. We believe that the origin of this defect is the difficulties which these quark models have in providing a sufficiently strong spin-orbit force for describing the splitting of the spin-triplet $P$ waves $\left({ }^{3} P_{0},{ }^{3} P_{1},{ }^{3} P_{2}\right)$ [12]. These spin-orbit forces are either generated by onegluon exchange and/or by the $\sigma$ exchange between quarks. Since the spin-orbit force provided by the one-gluon exchange is very weak [25] as compared to the central piece, one has to introduce a large coupling constant in order to achieve sufficient spin-orbit force, which, in turn, leads to a huge repulsive central contribution. Agreement with the experimental phase shifts can then only be achieved by introducing a likewise huge attractive central ( $\sigma$-like) piece that counterbalances this strong repulsion. Those two ingredients can be adjusted in such a way that they compensate very well for the lower partial waves. But this does not work anymore for the higher partial waves because of the different ranges involved in these contributions. On the other hand, if the spin-orbit force is generated by $\sigma$ exchange alone, this contribution has to be made stronger than in conventional meson-exchange models as well, because in the latter models 
one also gets an additional and significant contribution to the spin-orbit force from $\omega$ exchange. As pointed out already above, such contributions are left out in most quark models. We should mention, however, that in a recent work by the Kyoto-Niigata group vector mesons were incorporated explictly [27] and, indeed, a better overall agreement with the data could be achieved. In particular, it seems that now the higher partial waves are fairly well described [28].

For obvious reasons the free parameters in those quark models have been adjusted to give a good description of the lower (i.e., $S, P$, and $D$ ) partial waves. But this procedure automatically fixes the medium-range (or meson-exchange) part of the $N N$ force and, consequently, the predictions of those models for the higher partial waves. Our investigations have shown that the meson-exchange part of the quark models is not realistic yet but rather in conflict with present-day knowledge about the medium- and long-range properties of the $N N$ force obtained from other sources. Thus, we confirm a conjecture that was already raised in Ref. [14]. At the same time we want to emphasize, however, that one should be careful with the second part of the conjecture stated in Ref. [14], namely, that the failure in describing the higher partial waves is not caused by a problem in the short-range part (i.e., the part of the $N N$ interaction that depends on the quark degrees of freedom), for the following reason: Low partial waves like $S$ waves feel the short-range part of the $N N$ interaction as well as the medium- and long-range parts. Thus, if the short-range part of the $N N$ force derived in those quark models still has deficiencies, it might be possible to conceal those at the expense of introducing large and unrealistic medium-range components into the $N N$ model in a more-orless phenomenological way. Of course, then these deficiencies will show up indirectly and somewhere else, namely, in unrealistic predictions for the higher partial waves.

At this point let us emphasize that we do not want to suggest by our analysis that boson exchange is the only and unique way to describe the medium-range component of the
$N N$ force. There is, in principle, no problem if the effective meson-exchange piece used in quark models is different from the one-boson-exchange potentials, say. The former incorporates effects beyond a simple ansatz for quark confinement and OGE forces in the quark model and thus it is natural to expect such differences. However, the various effective meson-exchange potentials employed in the quark models that we have examined do not describe properly the higher partial waves. Thus, it seems that some medium-range pieces are still missing there. Indeed, in the context of quark models, there are additional sources for medium-range forces. E.g., a medium-range attraction can be generated by a quark delocalization mechanism, as demonstrated by the work of Goldman and collaborators [29].

In conclusion we believe that one should include our knowledge on the medium- and long-range parts of the $N N$ interaction from the very beginning and use it as a constraint for the $N N$ model to be constructed. Reliable results for the $N N$ interaction at intermediate ranges have been derived in the past, for example, from dispersion theory [19], as well as in an extended meson-exchange model [1] and more recently in the context of chiral perturbation theory [30,31]. These pieces of information should be utilized and supplemented with the short-range piece of the $N N$ interaction as it emerges from the quark-model picture. We believe that only by following this procedure can solid and conclusive results about the quality and reliability of a quark-model description of the short-range part of the $N N$ interaction be achieved.

\section{ACKNOWLEDGMENTS}

The authors thank Dr. Y. Fujiwara for sending us the phase shifts of the KYO-NII potential. Private discussions with A. Faessler, F. Fernández, A. Buchmann, and A. Valcarce are acknowledged. Financial support for this work was provided in part by the international exchange program DLR (Germany, BRA W0B 2F)-CNPq (Brazil, 910133/94-8).
[1] R. Machleidt, K. Holinde, and Ch. Elster, Phys. Rep. 149, 1 (1987).

[2] R. Machleidt, Adv. Nucl. Phys. 19, 189 (1988).

[3] For a list of references, see A. Le Yaouanc, Ll. Oliver, O. Pène, and J.-C. Raynal, Hadron Transitions in the Quark Model (Gordon and Breach, New York, 1988).

[4] D. A. Liberman, Phys. Rev. D 16, 1542 (1977).

[5] V. G. Neudatchin, Y. F. Smirnov, and R. Tamagaki, Prog. Theor. Phys. 58, 1072 (1977); I. T. Obukhovsky, V. G. Neudatchin, Y. F. Smirnov, and Y. M. Tchuvil'sky, Phys. Lett. 88B, 231 (1979).

[6] M. Harvey, Nucl. Phys. A352, 326 (1981).

[7] J. E. F. T. Ribeiro, Z. Phys. C 5, 27 (1980).

[8] C. S. Warke and R. Shanker, Phys. Rev. C 21, 2643 (1980).

[9] M. Oka and K. Yazaki, Phys. Lett. 90B, 41 (1980); Prog. Theor. Phys. 66, 551 (1981); 66, 5572 (1981).

[10] A. Faessler, F. Fernandez, G. Lübeck, and K. Shimizu, Phys. Lett. 112B, 201 (1982); Nucl. Phys. A402, 555 (1983).
[11] F. Fernández, A. Valcarce, U. Straub, and A. Faessler, J. Phys. G 19, 2013 (1993); A. Valcarce, A. Buchmann, F. Fernández, and A. Faessler, Phys. Rev. C 50, 2246 (1994).

[12] A. Valcarce, A. Buchmann, F. Fernández, and A. Faessler, Phys. Rev. C 51, 1480 (1995).

[13] A. Valcarce, P. González, F. Fernández, and V. Vento, Phys. Lett. B 367, 35 (1996); A. Valcarce, F. Fernández, and P. González, Phys. Rev. C 56, 3026 (1997); Few-Body Syst., Suppl. 10, 395 (1999); D. R. Entem, F. Fernández, and A. Valcarce, Phys. Rev. C 62, 034002 (2000).

[14] S. Takeuchi, K. Shimizu, and K. Yazaki, Nucl. Phys. A504, 777 (1989).

[15] Y. Fujiwara, C. Nakamoto, and Y. Suzuki, Phys. Rev. Lett. 76, 2242 (1996); Phys. Rev. C 54, 2180 (1996).

[16] K. Yazaki, Prog. Part. Nucl. Phys. 24, 353 (1990).

[17] Y. Fujiwara and K. T. Hecht, Nucl. Phys. A451, 625 (1986).

[18] R. Vinh Mau, C. Semay, B. Loiseau, and M. Lacombe, Phys. Rev. Lett. 67, 1392 (1991). 
[19] M. Lacombe, B. Loiseau, J. M. Richard, R. Vinh Mau, J. Coté, P. Pires, and R. de Tourreil, Phys. Rev. C 21, 861 (1980).

[20] V. G. J. Stoks, R. A. M. Klomp, C. P. F. Terheggen, and J. J. de Swart, Phys. Rev. C 49, 2950 (1994).

[21] R. Machleidt, Phys. Rev. C 63, 024001 (2001).

[22] V. G. J. Stoks, R. A. M. Klomp, M. C. M. Rentmeester, and J. J. de Swart, Phys. Rev. C 48, 792 (1993).

[23] R. A. Arndt, J. S. Hyslop III, and L. D. Roper, Phys. Rev. D 35, 128 (1987).

[24] D. V. Bugg and R. A. Bryan, Nucl. Phys. A540, 449 (1992).

[25] Note that the actual treatment of the OGE spin-orbit force in the quark model is still under dispute. See, e.g., Y. Fujiwara and K. T. Hecht, Nucl. Phys. A456, 669 (1986); Y. Fujiwara, M. Kohno, T. Fujita, C. Nakamoto, and Y. Suzuki, ibid. A674, 493 (2000).

[26] K. Holinde, Nucl. Phys. A543, 143c (1992).
[27] Y. Fujiwara, T. Fujita, M. Kohno, C. Nakamoto, and Y. Suzuki, Few-Body Syst., Suppl. 12, 311 (2000).

[28] Y. Fujiwara (private communication).

[29] T. Goldman and G. J. Stephenson, Jr., Phys. Lett. 146B, 143 (1984); T. Goldman, G. J. Stephenson, Jr., and K. E. Shmidt, Nucl. Phys. A481, 621 (1988); Fang Wang, Guang-han Wu, Li-jian, and T. Goldman, Phys. Rev. Lett. 69, 2901 (1992); T. Goldman, K. Maltmann, and G. J. Stephenson, Jr., Phys. Lett. B 324, 1 (1994); Guang-han, Jia-Lun Ping, Li-jian Teng, Fan Wang, and T. Goldman, Nucl. Phys. A673, 279 (2000);

[30] M. R. Robilotta and C. A. da Rocha, Nucl. Phys. A615, 391 (1997); J.-L. Ballot, M. R. Robilotta, and C. A. da Rocha, Phys. Rev. C 57, 1574 (1998).

[31] N. Kaiser, R. Brockmann, and W. Weise, Nucl. Phys. A625, 758 (1997); N. Kaiser, S. Gerstendörfer, and W. Weise, ibid. A637, 395 (1998). 